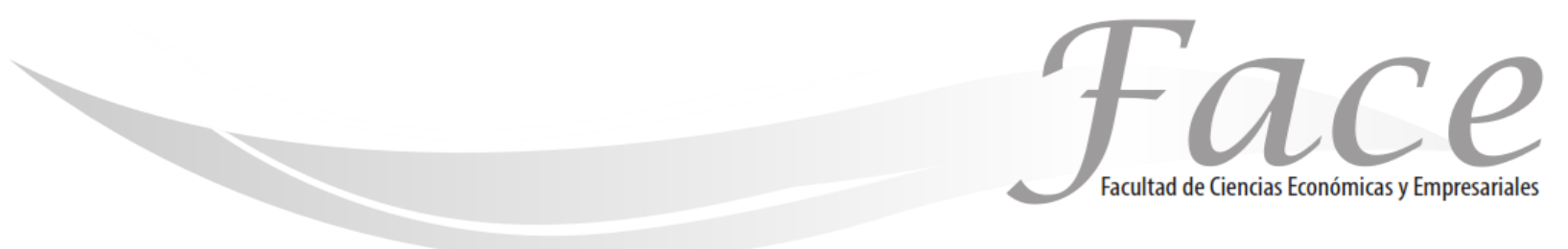

ISSN Impreso: 1794-9920

ISSN Electrónico: 2500-9338

Volumen 19-N²

Año 2019

Págs. 115 - 124

\title{
LA CULTURA DE SEGURIDAD DEL PACIENTE EN EL PERSONAL DE ENFERMERİA DEL HOSPITAL DE LA MUJER (SSA) EN PUEBLA
}

\author{
Mónica García Castilla * \\ ORCID: https://orcid.org/0000-0001-6405-3011 \\ Emigdio Larios-Gómez ** \\ ORCID: https://orcid.org/0000-0002-3514-1319
}

Fecha de Recepción: Julio 31 de 2019

Fecha de Aprobación: Octubre 1 de 2019

\section{Resumen:}

El objetivo de la investigación es analizar los factores de la Cultura de Seguridad del Paciente en el Personal de Enfermería del Hospital de la Mujer (SSA) Puebla, que permita definir acciones para mejorar la Cultura de Seguridad del Paciente. Se trata de un estudio Cuantitativo, descriptivo, transversal, y no experimental, realizado de octubre a noviembre 2017 en el Hospital de la Mujer de la SSA Puebla. La metodología de la aplicación del cuestionario sobre la Cultura de Seguridad del Paciente en Hospitales de México, Dirección General de Calidad y Educación en Salud (2013). Mismo que será utilizado en esta investigación, instrumento avalado por la Secretaria de Integración y Desarrollo del Sector Salud de la Dirección General de Calidad y Educación en Salud. Se realiza el cálculo de la muestra al 95\% de confianza, siendo un resultado para la aplicación del instrumento a 72 enfermeras de los diferentes turnos. Se realizó cada una de las dimensiones de la Cultura de Seguridad del Paciente en Hospitales de México, a través del sistema SPSS versión 21. Debido a que el personal, responde de manera negativa en cada una de las dimensiones, siendo lo siguiente: en su área de trabajo con un $40 \%$, en su supervisor/jefe con un $51 \%$, comunicación con un $30 \%$, frecuencia de eventos adversos reportados $36 \%$, concluyendo un puntaje de 32 en grado de seguridad aceptable, en su hospital cumple con el $44 \%$ de acuerdo, los resultados no se consideran una fortaleza debido a que ninguna de las dimensiones cubren mayor del $75 \%$ de las respuestas positivas los criterios no se cumplen, por lo tanto todas las dimensiones se consideran como "oportunidad de mejora".

Palabras clave: seguridad del paciente, hospitales, enfermería, cultura de seguridad.

* Doctorante en Alta Dirección. Universidad del Valle de Puebla, México. Contacto: monicagarciacruzroja@hotmail.com.

** Doctor en Ciencias Administrativas por el IPN ESCA Santo Tomas, Profesor-Investigador, Benemérita Universidad Autónoma de Puebla, México.Facultad de Administración, herr.larios@gmail.com 


\title{
THE CULTURE OF SECURITY OF THE PATIENT IN THE NURSING STAFF OF THE HOSPITAL DE LA MUJER (SSA) IN PUEBLA
}

\begin{abstract}
:
The objective of the research is to analyze the factors of the Safety Culture of the Patient in the Nursing Staff of the Hospital de la Mujer (SSA) Puebla, which allows defining actions to improve the Safety Culture of the Patient. This is a quantitative, descriptive, cross-sectional and non-experimental study, carried out from October to November 2017 at the Women's Hospital of SSA Puebla. Methodology of the application of the questionnaire on the Culture of Patient Safety in Hospitals of Mexico, General Directorate of Quality and Education in Health (2013). Same as will be used in this research, an instrument endorsed by the Secretary of Integration and Development of the Health Sector of the General Directorate of Quality and Health Education. The calculation of the sample is performed at $95 \%$ confidence, with 72 nurses from the different shifts being a result for the application of the instrument. Each of the dimensions of the Patient Safety Culture in Hospitals of Mexico was carried out through the SPSS version 21 system. Because the staff, responds negatively in each of the dimensions, being the following: in your area of work with $40 \%$, in your supervisor / boss with $51 \%$, communication with $30 \%$, frequency of events reported adverse $36 \%$, concluding a score of 32 in degree of acceptable safety, in your hospital meets the $44 \%$ agreement, the results are not considered a strength because none of the dimensions cover more than $75 \%$ of the positive responses the criteria are not met, therefore all dimensions are considered as "opportunity for improvement".
\end{abstract}

Keywords: patient safety, hospitals, nursing, safety culture.

\section{A CULTURA DA SEGURANÇA DO PACIENTE NO PESSOAL DE ENFERMAGEM DO HOSPITAL DE LA MUJER (SSA) EM PUEBLA}

\section{Resumo:}

O objetivo da investigação é analisar os fatores da Cultura de Segurança do Paciente no Pessoal de Enfermagem do Hospital da Mulher (SSA) Puebla, que permite definir ações para melhorar a Cultura de Segurança do Paciente. Se trata de um estúdio qualitativo, descritivo, transversal e não experimental, realizado em outubro de 2017 no Hospital da Mulher do SSA Puebla. A metodologia de aplicação do questionário sobre a Cultura de Segurança do Paciente nos Hospitais do México, Direção Geral de Qualidade e Educação em Saúde (2013). Ele será usado nesta investigação, instrumento avaliado pela Secretaria de Integração e Desenvolvimento do Setor de Saúde da Direção Geral de Qualidade e Educação em Saúde. Realize o cálculo da freqüência de $95 \%$ da confiança, seguindo um resultado para a aplicação do instrumento a 72 pacientes de diferentes turnos. Se você realiza uma das dimensões da Cultura de Segurança do Paciente nos Hospitais do México, com acesso ao sistema SPSS versão 21. Debido no que é pessoal, responde com a negativa negativa em uma das dimensões, seguindo o seguinte: área de trabalho com $40 \%$, no supervisor / gerente com $51 \%$, comunicação com 30\%, frequência de eventos adversos relatados $36 \%$, conclusão de 32 pontos na classificação de segurança aceitável, no total de hospitais $44 \%$ de desconto, os resultados não consideram uma fortaleza debitada na lista de dimensões máximas de $75 \%$ das respostas positivas a critérios não cumplen, para todas as dimensões consideradas como "oportunidade de aproveitamento".

Palavras-chave: segurança do paciente, hospitais, enfermagem, cultura de segurança. 


\section{ANTECEDENTES:}

La seguridad del paciente desde la perspectiva de la atención de salud supone un conjunto de normas, procedimientos, instrumentos y métodos basados en evidencias científicas destinadas a minimizar el riesgo y el daño sobre agregado y de eventos adversos en atención de salud. Se pretende garantizar las prácticas terapéuticas y de cuidados de enfermería seguros, así como de ajuste al medio ambiente, organización y funcionamiento institucional, incluyendo dotación de recurso humano y sostenimiento de personal. La Organización Mundial de la Salud (OMS, 2011) en relación con la calidad de la atención y seguridad del paciente establece, que las intervenciones de atención a la salud se realizan con el propósito de beneficiar al paciente, pero también pueden causarle daño.

Meléndez et al (2014) afirman que la seguridad del paciente debe ser considerada como prioritaria en los servicios de salud, ya que en la asistencia sanitaria actual se entrelazan procesos, tecnologías e interacciones humanas que al ser más complejas involucran nuevos riesgos adicionales y potenciales tanto para los pacientes como para los trabajadores del área. Es por ello la importancia de estos aspectos que se encuentra en las distintas declaraciones planes y programas promovidos por Organización Mundial de la salud (OMS) para afrontar el problema de la seguridad de los pacientes, que es entendida como estructuras y procesos organizacionales que disminuyen la posibilidad de padecer efectos adversos como consecuencia de la atención recibida en una Institución de salud.

La Preocupación de la seguridad del paciente surge de la observación de la Incidencia de eventos adversos que impacta negativamente en la Calidad de atención, es por ello por lo que la mayor parte del tiempo que permanece el paciente directamente en su atención y cuidado en área hospitalaria es a cargo del Personal de Enfermería, por lo tanto, es importante intervenir en la generación de conocimiento que permitirá inferir en la evaluación de la Cultura de la Seguridad del paciente.
Estudio a Nivel Nacional de Efectos Adversos (2015-2020): La Seguridad del Paciente, dimensión esencial de la calidad implica desarrollar estrategias para reducir el daño innecesario al paciente asociado a la estancia sanitaria, la Organización Mundial de la Salud lanzó en el 2004, la Alianza por la Seguridad del paciente promoviendo acciones, herramientas y recomendaciones para mejorar la seguridad en todos los países del mundo. Los objetivos de la estrategia han estado básicamente orientados a mejorar la cultura de la seguridad y la gestión del riesgo sanitario, la formación de los profesionales, la implementación de prácticas seguras, la implicación de los pacientes y ciudadanos y la participación internacional, Los estudios epidemiológicos desarrollados en el marco de esta estrategia y los datos de evaluación de los programas y acciones impulsados a nivel nacional y regional permiten tener un conocimiento válido y preciso del nivel de seguridad del paciente. A continuación, se presenta estudio Nacional de 42 hospitales españoles. (Tabla 1). Ver siguiente página.

Por lo tanto, la Organización Mundial de la Salud, encamino a los países y miembros a trabajar en el tema para evitar eventos adversos previsibles, en el 2004 la encuesta Nacional hecha por la agencia de mejoramiento de calidad en salud (AHRQ). El primer estudio que en Latinoamérica evidenció el problema de la seguridad fue el estudio IBEAS. Hecho en 5 países de la región (México, Costa rica, Colombia, Perú y Argentina) evidencio que, en estos países, en promedio 1 de cada 10 pacientes hospitalizados (10\%) sufren al menos un daño durante su atención, la OMS considera que el concepto de calidad en servicios de salud debe de estar en un alto nivel de excelencia profesional uso eficiente de recursos, mínimo riesgo para el paciente, alto riesgo de satisfacción por parte del paciente en un impacto final de salud (Ruiz y Montes, 2012). 
Tabla 1,

Principales datos de Estudio Nacional de Eventos Adversos Relacionados con la Hospitalización.

\begin{tabular}{|c|c|c|c|}
\hline Tipos de Eventos Adversos & $\mathbf{N}$ & $\%$ & Evitable \\
\hline Relacionados con los cuidados & 50 & 7,63 & 56,0 \\
\hline Úlcera por presión & 24 & 3,66 & \\
\hline $\begin{array}{l}\text { Quemaduras, erosiones y contusiones } \\
\text { (incluyendo fracturas consecuencias) }\end{array}$ & 19 & 2,90 & \\
\hline Enfisema agudo de pulmón e Insuficiencia respiratoria & 4 & 0.61 & \\
\hline Otras consecuencias de la inmovilización prolongada & 3 & 0.46 & \\
\hline Relacionado con la Medicación & 245 & 37,4 & 34,8 \\
\hline Náuseas, vómitos o diarrea secundarios a medicación & 32 & 4,89 & \\
\hline Prurito, rash o lesiones dérmicas reactivas a fármacos o apósitos & 32 & 4,89 & \\
\hline Otros efectos secundarios de fármacos & 29 & 4,43 & \\
\hline Mal control de la glucemia & 19 & 2,90 & \\
\hline Hemorragia por anticoagulación & 18 & 2,75 & \\
\hline Otros & 104 & 15,98 & \\
\hline Relacionado con la Infección Nosocomial & 166 & 25,34 & 56,6 \\
\hline Infección de herida quirúrgica & 50 & 7,63 & \\
\hline Infección tracto urinario nosocomial & 45 & 6,87 & \\
\hline $\begin{array}{l}\text { Otro tipo de infección nosocomial o infección nosocomial sin } \\
\text { especificar }\end{array}$ & 22 & 3,36 & \\
\hline Sepsis y shock séptico & 19 & 2,90 & \\
\hline Neumonía nosocomial & 17 & 2,60 & \\
\hline Bacteriemia asociada a dispositivo & 13 & 1,98 & \\
\hline Relacionados con un procedimiento & 164 & 25,04 & 31,7 \\
\hline $\begin{array}{l}\text { Hemorragia o hematoma relacionadas con intervención quirúrgica o } \\
\text { procedimiento }\end{array}$ & 61 & 9,31 & \\
\hline Lesión en un órgano durante un procedimiento & 20 & 3,05 & \\
\hline Otras complicaciones tras intervención quirúrgica o procedimiento & 14 & 2,14 & \\
\hline Intervención quirúrgica ineficaz o incompleta & 11 & 1,68 & \\
\hline Desgarro uterino & 9 & 1,37 & \\
\hline Otros & 40 & 7,48 & \\
\hline Relacionado con el Diagnostico & 18 & 2,75 & 84,2 \\
\hline Retraso en el diagnóstico & 10 & 1,53 & \\
\hline Error diagnóstico & 8 & 1,22 & \\
\hline Otros & 12 & 1,83 & 33,4 \\
\hline Pendiente de especificar & 7 & 1.07 & \\
\hline Otros eventos adversos & 5 & 0,76 & \\
\hline Total & 655 & 100,00 & 42,6 \\
\hline
\end{tabular}

Fuente: Estrategia de Seguridad del Paciente del Sistema Nacional de Salud (2015-2020)

Metas Internacionales de Seguridad del Paciente

La Seguridad del Paciente toma como relevancia, a partir de la detección de efectos adversos derivado de la atención Médica en pacientes hospitalizados en la Ciudad de Nueva York, estados unidos en 1984, (Harvard Medical Practice Study encontrando incidencia de efectos adversos $70 \%$ discapacidad moderada, $2.6 \%$ permanente, $13.6 \%$ provoco muerte, en 1999 el Instituto de Medicina de Estados Unidos publico informe de "To Err is Human: Building a Safer Health System" sugiriendo que el $50 \%$ de los eventos adversos en Nueva York y Colorado y Utha pueden ser previsibles, es por ello que la seguridad del paciente se define como el con junto de estructuras y procesos y resultados organizacionales que reducen al máximo y previene la probabilidad de sufrir un evento adverso durante la atención de salud de los usuarios, las alianzas entre los hospitales destinadas a mejorar la seguridad del paciente mediante la implantación de objetivos y acciones de mejora basados en 6 metas internacionales como solución para los sistemas de salud mundiales, que fueron publicadas por la Organización Mundial de la Salud desde el 2001, dadas a la comunidad internacional continuando vigentes, mencionando las siguientes, (DGCS, 2016), ver Tabla 2. 
Tabla 2.

Estructura de Objetivos Prioritarios de Metas Internacionales de Seguridad del Paciente

\begin{tabular}{ll}
\hline $\begin{array}{l}\text { Metas Internacionales de Seguridad } \\
\text { del Paciente (MISP) }\end{array}$ & Objetivos Prioritarios \\
\hline $\begin{array}{l}\text { 1.- Identificar correctamente a los } \\
\text { pacientes }\end{array}$ & $\begin{array}{l}\text { Mejorar la precisión en la identificación } \\
\text { de los pacientes para prevenir errores } \\
\text { que involucran al paciente equivocado. }\end{array}$
\end{tabular}

2.- Mejorara la comunicación afectiva Prevenir errores que involucren el tipo de comunicación más propensa a la equivocación, ordenes y/o información de resultados críticos: verbales y/o telefónica.

3.-Mejorar la Seguridad de los Prevenir la administración errónea de Analiza la inclusión de medicamentos Medicamentos de Alto Riesgo medicamentos de ALTO RIESGO de alto riesgo aunado a lo establecido de acuerdo al análisis de riesgos institucionales. 4.- Garantizar cirugías en el lugar
correcto, con el procedimiento con el procedimiento incorrecto y la
correcto, con el procedimiento persona equivocada correcto y al paciente correcto.

5.- Reducir el Riesgo de Infecciones Prevenir y reducir el riesgo de asociadas a la atención médica
Prevenir y reducir el riesgo de
infecciones mediante la adecuada higiene de manos

En el 2015 se Actualiza la Cédula de Acreditación con Mayor Enfoque a la Seguridad del Paciente

Generar una inclusión total de los pacientes en su seguridad a través de la actualización, aplicación y supervisión del procedimiento para su adecuada identificación.

Incluir el aviso de alerta para resultados de laboratorio, gabinete y patología fuera de los rasgos establecidos

Vigilar y fortalecer el procedimiento para el correcto llenado de la lista de verificación para la seguridad de la cirugía.

Incluir en el INDICAS un índice específico para la prevención de infecciones asociadas a la atención de salud, en acuerdo con el instituto nacional de ciencias médicas $y$ nutricionales Salvador Zubirán y la AMEIN

\begin{tabular}{|c|c|c|}
\hline $\begin{array}{l}\text { 6.- Reducir el Riesgo de daño al } \\
\text { paciente por causa de caídas }\end{array}$ & $\begin{array}{l}\text { Prevenir y reducir el riesgo de caídas en } \\
\text { los pacientes hospitalizados, prevenir y } \\
\text { reducir el riesgo de caída en los } \\
\text { pacientes hospitalizados }\end{array}$ & $\begin{array}{l}\text { Fortalecer la autogestión justo con el } \\
\text { paciente y/o cuidador primario, para } \\
\text { evitar caídas y/o lesiones por una } \\
\text { inadecuada valoración y revaloración } \\
\text { del estado de salud, así como una } \\
\text { mayor difusión y capacitación conjunta. }\end{array}$ \\
\hline
\end{tabular}

Fuente: Dirección General de Calidad y Educación en Salud (2016) y Manual de políticas Instituciones de salud (2015).

\section{Acciones Esenciales para la Seguridad del Paciente (2017)}

La seguridad para el paciente durante los procesos de atención durante las estimaciones en México el $2 \%$ de pacientes hospitalizados muere y el $8 \%$ padece algún daño a causa de eventos adversos, relacionados con la seguridad, sin embargo, el $62 \%$ los eventos son previsibles, para atender esta problemática determinando ciertas estrategias. Una de las funciones estratégicas del Sistema Nacional de certificación de establecimientos de Atención médica (SiNaCEAM), y del equipo colaborativo con la Dirección General de Calidad y Educación en Salud, surge el desarrollo y publicación conjunta a las Acciones Esenciales para la Seguridad del Paciente (AESP), primer paso hacia el Modelo de Complementación AcreditaciònCertificaciòn. Consejo de Salud General (CSG -2017).

\section{Estándares para Implementar el Modelo en Hospitales} (2018).

El Modelo de seguridad del paciente del SiNaCEAM, se diseñó para implementar los estándares basados en diseños e implementación de procesos seguros, ayudando a prevenir que sucedan eventos adversos 0 eventos centinelas, el Modelo fomenta en las organizaciones el desarrollo de la Cultura de Calidad y Seguridad del paciente, en la que se aprende del error y se evite al máximo la ocurrencia de este evento, implementando barreras de seguridad estandarizadas.

A continuación, se definen las 8 acciones Esenciales para la Seguridad del Paciente. Estándares para Implementar el Modelo de Hospitales (2018). Tabla 3 
Tabla 3.

Acciones Esenciales para la Seguridad del Paciente

\begin{tabular}{|c|c|c|}
\hline No & ACCIÒN & CONCEPTO \\
\hline 1 & AESP.1 Identificación Correcta del Paciente & $\begin{array}{l}\text { Es el uso, de al menos dos de los datos para identificar a un } \\
\text { paciente. (nombre del paciente, número de identificación, fecha de } \\
\text { nacimiento). }\end{array}$ \\
\hline 2 & AESP.2 Comunicación Efectiva & $\begin{array}{l}\text { La comunicación puede ser electrónica, oral o escrita, las } \\
\text { comunicaciones más propensas al error son las verbales, es por } \\
\text { ello importante al dar las órdenes el proceso de escuchar, escribir, } \\
\text { leer y confirmar. }\end{array}$ \\
\hline 3 & $\begin{array}{l}\text { AESP.3 Seguridad en el Proceso de } \\
\text { Medicación }\end{array}$ & $\begin{array}{l}\text { Los medicamentos forman parte del tratamiento de un paciente, y } \\
\text { es fundamental manejarlos de manera adecuada con el fin de } \\
\text { garantizar la seguridad (Electrolitos concentrados, quimioterapia, } \\
\text { radiofármacos, insulinas, anticoagulantes por vía parenteral, } \\
\text { producen mayor eventos centinelas) }\end{array}$ \\
\hline 4 & AESP.4 Seguridad de los Procedimientos & $\begin{array}{l}\text { El procedimiento es disminuir la probabilidad de que ocurran } \\
\text { eventos adversos, centinelas relacionados con realización } \\
\text { incorrecta de un procedimiento, a través del protocolo universal } \\
\text { (marcado en el sitio anatómico, proceso de verificación pre- } \\
\text { procedimiento, tiempo fuera) }\end{array}$ \\
\hline 5 & $\begin{array}{l}\text { AESP.5 Reducción del Riesgo de Infecciones } \\
\text { Asociadas A Atención de la Salud }\end{array}$ & $\begin{array}{l}\text { Este proceso es a través de higiene adecuada de manos, el cual se } \\
\text { basa en la adaptación o adopción de los lineamientos vigentes, } \\
\text { para higiene de manos como establece la (OMS). }\end{array}$ \\
\hline 6 & $\begin{array}{l}\text { AESP. } 6 \text { Reducción del Riesgo de Daño al } \\
\text { Paciente por Caída. }\end{array}$ & $\begin{array}{l}\text { Es una de las causas más comunes de lesiones y daños al } \\
\text { paciente, en el contexto de la población que atiende, los servicios } \\
\text { que presta y las características de las instalaciones de las } \\
\text { organizaciones, debe evaluar el riesgo de caída para reducir esta } \\
\text { probabilidad. }\end{array}$ \\
\hline 7 & $\begin{array}{l}\text { AESP. } 7 \text { Registros y Análisis de Eventos } \\
\text { Centinela, Eventos Adversos y Cuasi fallas. }\end{array}$ & $\begin{array}{l}\text { Generar información sobre cuasi fallas, eventos adversos y } \\
\text { centinelas, mediante una herramienta de registro que permita el } \\
\text { análisis y favorezca la toma de decisiones para que a nivel local se } \\
\text { prevenga su ocurrencia. }\end{array}$ \\
\hline 8 & AESP. 8 cultura de Seguridad del Paciente & $\begin{array}{l}\text { Medir la Cultura de Seguridad del paciente en el ámbito } \\
\text { hospitalario, con el propósito de favorecer la toma de decisiones, } \\
\text { para establecer acciones de mejora continua del clima de seguridad } \\
\text { en los hospitales del sistema Nacional de Salud. }\end{array}$ \\
\hline
\end{tabular}

Fuente: Acciones Esenciales para seguridad del paciente, dentro el Modelo de Seguridad del Paciente de Consejo de Salud General (CSG -2017). Estándares para Implementar el Modelo de Hospitales (2018). 
Dando seguimiento a lo mencionado es importante definir las Metas Internacionales para dar seguimiento a la Seguridad del paciente a las Instituciones de Salud. Con la intención de tener la información y disminuir los riesgos de incidentes, omisiones y efectos adversos, y así mismo la investigación ayudará a definir las acciones en la Cultura que tiene el personal Operativo de enfermería del Hospital de la Mujer sobre la Seguridad del paciente respaldándonos a través del Instrumento de la Metodología de aplicación del cuestionario sobre cultura de seguridad del paciente en Hospitales de México (2012), con la intensión de identificar las debilidades y convertirlas en fortalezas e intervenir de manera oportuna, siendo que esta investigación formará parte de la evidencia que contribuirá a futuros proyectos para identificar los posibles riesgos y tomar decisiones oportunas para un aporte Institucional para seguridad del paciente, trabajador y de la Organización, teniendo un impacto en la toma de decisiones para el "Doctorado en Alta Dirección" y poder intervenir en decisiones oportunas para mejorar la Cultura del Personal de Enfermería, y se vea reflejado en la atención directa al paciente, disminuyendo los riesgos e incidentes.

En el Hospital de la Mujer de los Servicios de Salud del Estado de Puebla no se exime que se presenten factores de riesgo en la atención al paciente, siendo que se han presentado diversos incidentes en la atención directa, generando como resultado efectos adversos y cuasi fallas debido a diferentes factores, mencionando que estas situación puede ser previsible evitando llegar a un efecto centinela, siempre y cuando se identifique que factores contribuyen a que se genere esta condición de inseguridad, por lo tanto es importante poder intervenir en momento oportuno disminuyendo riesgos, daños, y repercusiones para el paciente, personal de enfermería y la organización.

La preocupación de la seguridad del paciente surge de la observación de la Incidencia de eventos adversos que impacta negativamente en la Calidad de atención, es por lo que la mayor parte del tiempo que permanece el paciente directamente en su atención y cuidado en área hospitalaria es a cargo del Personal de Enfermería y el área Médica, por lo tanto, es importante intervenir en la generación de conocimiento que permitirá inferir en la evaluación de la Cultura de la Seguridad del paciente.

Con la intención de tener información y disminuir los riesgos de incidentes, omisiones y efectos adversos, y así mismo nos ayudará a definir las acciones para mejorar la Cultura que tiene el personal operativo de enfermería del Hospital de la Mujer sobre la Seguridad del paciente respaldándonos a través de la aplicación del cuestionario de cultura de seguridad del paciente con la intensión de identificar las debilidades y convertirlas en fortalezas e intervenir de manera oportuna, siendo que esta investigación formará parte de la evidencia que contribuirá a futuros proyectos de intervención en identificar los posibles riesgos y tomar decisiones oportunas para un aporte Institucional para seguridad del Paciente, trabajador y de la Organización. Es por lo que surge la siguiente interrogante ¿Cuáles son los factores que predominan en el personal de Enfermería en relación de la Cultura de Seguridad del Paciente?

\section{MATERIALES Y MÉTODOS :}

El objetivo de la investigación es analizar los factores de la Cultura de Seguridad del Paciente en el Personal de Enfermería del Hospital de la Mujer (SSA) Puebla, que permita definir acciones para mejorar la Cultura de Seguridad del Paciente.

Se trata de un estudio Cuantitativo, descriptivo, transversal, y no experimental, realizado de octubre a noviembre 2017 en el Hospital de la Mujer de la SSA Puebla. a metodología de la aplicación del cuestionario sobre la Cultura de Seguridad del Paciente en Hospitales de México, Dirección General de Calidad y Educación en Salud (2013). Mismo que será utilizado en esta investigación, instrumento avalado por la Secretaria de Integración y Desarrollo del Sector Salud de la Dirección General de Calidad y Educación en Salud, este cuestionario fue diseñado por la Agency For Healthcare Research and Quality, agencia para la calidad e investigación en servicios de Salud, En los Estados Unidos bajo el nombre de Hospital Survey con Patient Safety Culture En México esta herramienta es el resultado de un proceso de adaptación al personal de salud, que consistió en una aplicación piloto de 12 hospitales públicos del sector salud en el 2009, está compuesto por 53 preguntas agrupadas en 8 secciones, 42 de las cuales se relacionan con 12 dimensiones de la cultura de la seguridad del paciente y las restantes con la información general del encuestado.

Se entrevistó al Personal de Enfermería operativo de los diferentes turnos, adscrito al hospital de la Mujer de los diferentes servicios clínicos, que están en contacto directo con pacientes. Se incluyen en esta categoría personal con código de Enfermera general titulada, Enfermera especialista titulada y auxiliar de enfermería. El tamaño de la muestra se realizó a través de la tabla establecida en el instrumento de Dirección General de Calidad y Educación en Salud correspondiente. Se realiza el cálculo de la muestra al $95 \%$ de confianza, siendo un resultado para la aplicación del instrumento a 72 enfermeras de los diferentes turnos. (Tabla 4). 
concluyendo que mi supervisor/jefe considera seriamente la sugerencia del personal para mejorar la seguridad de los pacientes.

Tabla 4.

Total de personal de enfermería respecto al tamaño de muestra

\begin{tabular}{|c|c|}
\hline Total, de personal operativo de enfermeria & $\begin{array}{c}\text { Tamaño de } \\
\text { muestra }\end{array}$ \\
\hline $83-85$ & 45 \\
\hline $86-89$ & 46 \\
\hline $90-93$ & 47 \\
\hline $94-96$ & 48 \\
\hline $97-119$ & 53 \\
\hline $120-124$ & 54 \\
\hline $125-130$ & 55 \\
\hline $131-135$ & 56 \\
\hline $136-141$ & 57 \\
\hline $142-148$ & 58 \\
\hline $149-154$ & 59 \\
\hline $155-161$ & 60 \\
\hline $162-169$ & 61 \\
\hline $170-179$ & 62 \\
\hline $180-189$ & 63 \\
\hline $190-199$ & 64 \\
\hline $200-209$ & 65 \\
\hline $210-219$ & 66 \\
\hline $220-229$ & 67 \\
\hline $230-239$ & 68 \\
\hline $240-259$ & 69 \\
\hline $260-269$ & 70 \\
\hline $270-289$ & 71 \\
\hline $290-299$ & 72 \\
\hline $300-319$ & 73 \\
\hline
\end{tabular}

Fuente: Dirección General de Calidad y Educación en Salud (2015).

\section{ANÁLISIS DE LOS RESULTADOS:}

Se realizó cada una de las dimensiones de la Cultura de Seguridad del Paciente en Hospitales de México, a través del sistema SPSS versión 21:

- De acuerdo con las 18 preguntas de la dimensión uno, se identifica que la pregunta 2 muestra una media de 1.9 siendo baja, concluyendo que el personal no tiene suficiente personal para enfrentar la carga de trabajo. Y la pregunta 4 fue la más alta con una media de 4.09 concluyendo que en mi área o servicio nos tratamos todo con respeto.

- De acuerdo con las 4 preguntas de la dimensión dos, se identifica que la pregunta 4 muestra una media de 2.4 siendo baja, concluyendo que el personal responde que $\mathrm{Mi}$ supervisor/ jefe no hace caso de los problemas de seguridad del paciente que ocurre una y otra vez. Y la pregunta 2 fue la más alta con una media de 3.54
- De acuerdo con las 6 preguntas de la dimensión tres, se identifica que la pregunta 4 muestra una media de 2.59 siendo baja, concluyendo que el personal no puede cuestionar con total libertad las decisiones o acciones de sus superiores. Y la pregunta 2 fue la más alta con una media de 3.37 concluyendo que el personal habla con libertad si ve algo que pueda afectar negativamente con la atención del paciente.

- De acuerdo con las 3 preguntas de la dimensión cuatro, se identifica que la pregunta 2 muestra una media de 3.44 siendo baja, concluyendo que cuando se comete un error que se estima que no puede dañar al paciente, ¿Qué tan frecuente se reporta respondiendo que se reporta a veces y a veces no. Y la pregunta 3 fue la más alta con una media de 3.79 concluyendo que cuando se comete un error que se estima podría haber dañado al paciente, pero no lo daño ¿Qué tan frecuente lo reporta, respondiendo la mayoría de las veces. 
- De acuerdo con las 5 puntuaciones de la dimensión cinco, se identifica que la puntuación 1 muestra una frecuencia en grado de seguridad del paciente de 2 siendo baja con resultado de excelente, la puntuación 3 muestra una frecuencia de grado de seguridad de paciente alta de 32 concluyendo que es Aceptable.

- De acuerdo con las 11 preguntas de la dimensión seis, se identifica que la pregunta 6 muestra una media de 2.36 siendo baja, concluyendo que con frecuencia es desagradable trabajar con personal de otros departamentos del hospital, $Y$ la pregunta 10 fue la más alta con una media de 3.52 concluyendo que los departamentos del hospital trabajan bien juntos para proporcionar el mejor cuidado a los pacientes.

\section{CONCLUSIONES}

Debido a que el personal, responde de manera negativa en cada una de las dimensiones, siendo lo siguiente: en su área de trabajo con un $40 \%$, en su supervisor/jefe con un $51 \%$, comunicación con un $30 \%$, frecuencia de eventos adversos reportados $36 \%$, concluyendo un puntaje de 32 en grado de seguridad aceptable, en su hospital cumple con el $44 \%$ de acuerdo, los resultados no se consideran una fortaleza debido a que ninguna de las dimensiones cubren mayor del $75 \%$ de las respuestas positivas los criterios no se cumplen, por lo tanto todas las dimensiones se consideran como "oportunidad de mejora".

En base a los resultados obtenidos no se encuentran las dimensiones en niveles positivos, ya que ninguna dimensión cubre el $75 \%$ de sus respuestas. Encontrando en respuesta negativa la dimensión de Su área de Trabajo, no se cuenta con suficiente personal para enfrentar la carga de trabajo con un $40 \%$ coincidiendo con Schonke et al (2016), Saturno, Dasilva, Olveira y Fonseca (2008), Acevedo, Castañeda, González y Pineda (2013), Jaraba, Sartolo, Villaverde, Espuis y Rivas (2013) con 68.1\%, Castañeda et al (2013) con 50\%, Fajardo et al (2010), no refleja porcentajes, Gómez et al (2009), Pérez et al (2014) con $32.7 \%$, Tomas, Chavanovas, Roqueta y Tarazo (2012), con $57 \%$, Hernández et al (2013), con $49 \%$, por lo tanto se concluye que es un riesgo laboral para el personal la seguridad del paciente.

En Comunicación muestra un 30\%, que el personal no puede cuestionar con libertad las decisiones o acciones de sus jefes, coincidiendo con, Fajardo et al (2010) con el $38 \%$. En Frecuencia de Eventos Adversos, no se reportan con un $38.9 \%$, coincidiendo con Ibarra, Olvera y Santacruz (2011) con un $58.1 \%$.
Se identifica que hay sensibilizar al personal operativo, inspirar confianza para que se puedan reportar los incidentes, realizar recomendaciones para mejorar, que identifique que la organización le apoyara para realizar mejor sus procesos, e insistir en la asistencia de la capacitación continua.

\section{BIBLIOGRAFÍA:}

Acevedo, P., Castañeda, H., Gonzàlez, S., y Pineda, Z., (2013). Percepciòn de la Cultura de la Seguridad del Paciente por el Personal de Enfermerìa. Ciencia y Enfermeria, 19(2), 77-88.

Modelo de seguridad del paciente dentro del consejo de salud general, edición (2017). Acciones Esenciales para la Seguridad del Paciente. Del sistema Nacional de certificación de establecimiento de atención médica (SiNaCEAM) pág. 13,32.

Agency for Healthcare Research and Quality (2016). Hsopital Survey on Patient Safety Culture: users Guide.

Estrategia de Seguridad del Paciente del Sistema Nacional de Salud (2015-2020). Ministerio de Sanidad, servicios sociales Igualdades. Pag 20.21.

Estándares para la implementación el Modelo en Hospitales, edición (2018) Sistema nacional de certificación de establecimiento en atención médica(SiNaCEAM). Pag,13,14.

Fajardo, D., Rodríguez, S., Arboleya, C., Rojano, F., Hernández, T., Santacruz, V., (2010) Cultura sobre Seguridad del Paciente en Profesional de Salud, Revista Científica de América Latina, Vol. 78, núm. 6, pág. 527-532.

Gomez, R., Arenas, E., Gonzàlez, V., Garzòn, S., Mateus, G., y Soto, G.,. (2009). Cultura de Seguridad del Paciente por personal de Enfermeria por Bogota. Revista Colombiana, 56-70.

Hernandez, M., Valle, A., Garcia, J., Lopera, Lopera., Luna, C., y Fernandez, B. (2013). Cultura de Seguridad del Paciente en el Servicio de Urgencias del Sistema AndaLuz de Salud. conamed, 18(4), 148-156. 
Ibarra, C; Olvera. S; Santacruz.V; (2011). Analisis de la Cultura de Seguridad del Paciente en el Hospital Regional de Alta Especialidad de Oaxaca. CONAMED, 16(2), 65-73.

Jaraba, B., Sartolo, R., Villaverde, R., Espuis, A., Rivas, R., (2013) Evaluación de Manual de politicas Institucionales de Calidad (2015). Metas internacionales de Seguridad del paciente, Rumbo a la certificación.

Martínez, T., Rojas, C., V., Zarate, G., Flores, M (2017) Florence Nightingale, pionera en el Modelo de la Calidad y seguridad del paciente, Revista, cuid Arte, el arte del cuidado, vol. 6, núm. 11, pág. 45. M, Soule (2014) Capitulo 1 Seguridad del paciente, pág. 1 http://theific.org/wpcontent/uploads/2014/08/Spanish_ch1_PRESS.p df

Melendez, M., Garza, H., Gonzalez, S., Castillo, C., Gonzalez, G., y Ruiz, H., (8 de mayo de 2014). Percepción del Personal de Enfermeria Hacia la Cultura de Seguridad en un Hospital Pediatrico en México. CUIDARTE, 5(3), 774-81.

Pérez, C., Castro, V., Jaramillo, E., Olvera, M., Burgos, M., Pastrana, González, O., Delgado, B., Márquez, V., Real, T., (2014). Percepción de la Cultura de Seguridad del Paciente en Médicos pasantes de servicio social. Consejo Nacional de Arbitraje Médico, 19 (2), pág. 52-58.

Politt, H., (2010) Libro de Investigaciòn cientifica en ciencias de la Salud. Sexta edición, editorial Mc Graw Hill, pág. 669.

Programa Nacional de Salud (2013), Programa Sectorial de Salud, primera edición, México D, F.

Rodríguez, L. A. J., \& Suárez, R. G. (2017). Gestion del Post-conflicto como estrategia de la responsabilidad social en las empresas. FACE: Revista de la Facultad de Ciencias Económicas y Empresariales, 17(1), 35-43.

Ruiz, V., Montes, Cruz., (2012). Seguridad del Paciente y Atenciòn Segura, guìa De buenas pràcticas para la Seguridad del Paciente en la atenciòn en salud, s/p.
Saturno, Z., Da Silva, G., Oliveira, S., Fonseca, A., (2008) Análisis sobre laCultura Sobre la seguridad del paciente en los hospitales del sistema nacional de salud, medicina clínica de Barcelona 131, (supl 3) pág. 18-25.

Schwonke, B., Camila, R., Lunardy, F., Wilson, D., Silva, S., Soares, y R.,Marcio, O., (2016). Cultura de seguridad: la percepción de los profesionales de Enferemeria Intensivista. Enfermeria Global, 2235.

Tomas, S., Chanovas, M., Roqueta, F., Toranzò, T., (2012). Seguridad del paciente en urgencias $y$ emergencias: balance de cuatro años del programa Semex-Seguridad del paciente, artículo de emergencias pág. 225-233. 